\title{
Effect of dissipation in rapid-gravitational granular flows
}

\author{
Yajuan Zhu ${ }^{1}$, Renaud Delannay ${ }^{1}$, and Alexandre Valance ${ }^{1, \star}$ \\ ${ }^{1}$ Univ Rennes, CNRS, Institut de Physique de Rennes, UMR 6251, 35042 Cedex Rennes, France
}

\begin{abstract}
We investigate numerically high speed granular flows down an incline and focus our attention on the influence of the restitution coefficient $e$ of binary collisions on the nature of the flow regimes. We show in particular that $e$ plays a major role in rapid flows. Decreasing $e$ leads in general to denser flows but also quicker flows. The increase of the mean flow velocity with decreasing $e$ is explained as the result of the clustering instability which produces a dense and cold core moving very fast as a plug.
\end{abstract}

\section{Introduction}

Recently, several works have been devoted to rapid granular flows confined between side walls. Due to side-wall frictional, steady and fully developed flows (SFD flows) have been observed up to large angles of inclination where accelerated ones are usually expected [1-4]. These new SFD regimes present non-trivial features, including secondary flows (rolls) and heterogeneous volume fraction. Among these, the supported flow regime is particularly interesting: It consists of a dense core floating over a dilute flowing layer and may have implications in the context of geophysical flows [2]. Very few experimental studies [58 ] were devoted to these high speed flow regimes and most of the knowledge comes from numerical simulations. Recent numerical works $[2,4]$ have explored extensively and systematically these different flow regimes as a function of the inclination angle $\theta$ and of the particle holdup $H$ for a given gap width $W=68 D$ between side walls.

Here we investigate flows with a narrower width $W=$ $20 D$ and a fixed particle hold-up $H=5 D$. We deal with rather small systems and thus have reasonable computing time to obtain SFD flows. This allows to conduct a parametric study to investigate the role of the restitution coefficient $e$ of binary collisions. We report here an unusual result which could be phrased as follows: the more dissipative the flows are, the faster they run.

\section{Simulation setup}

The simulation mimics a granular system flowing on a flat base and bounded by two side-walls. We used classical discrete element model (DEM). We considered spherical particles of mass $m$ with a mean diameter $D$ and slight poly-dispersity $( \pm 10 \% D)$. The motion of each individual particle is computed from forces acting on them, including contact forces with other particles and basal and side walls,

\footnotetext{
^e-mail: alexandre.valance@univ-rennes1.fr
}

A video is available at https://doi.org/10.48448/3gyw-ky42 and gravitational forces. Particle-particle and particle-wall interactions are described via a spring-dashpot model in both normal and tangential directions, including Coulomb friction. Details of the simulation method and parameters are given in [1,2]. Particle-particle and particle-wall friction coefficients are taken from experiments [9] and set to 0.33 and 0.593 , respectively. The normal restitution coefficient for the particle-wall collision is kept fixed and set to 0.8 , while the one for the particle-particle collision (referred later to as $e$ ) is varied between 1 and 0.55 . The latter is tuned by adjusting the dashpot constant while the spring stiffness is kept fixed and set to $10^{5}(\mathrm{mg} / \mathrm{D})$. The system length in the stream-wise direction is $L=20 D$ and the width is $W=20 D$. The system is inclined with an angle $\theta$ with respect to the horizontal. Periodic boundary conditions are used in the steam-wise direction (see Figure 1).

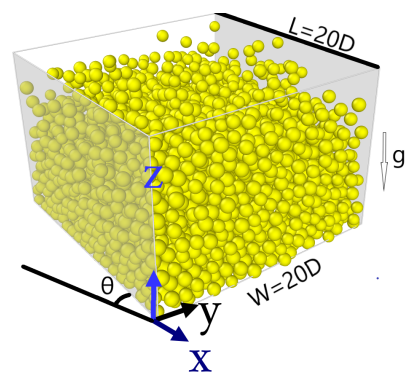

Figure 1: Flow geometry: The granular flow is confined by lateral walls with a width $W=20 D$. Periodic boundary conditions are used in the stream-wise direction with a length $L$ and an angle of inclination $\theta$.

Here we set the particle hold-up $H$ to a fixed value $H=5 D$. The particle hold up represents the depthintegrated particle volume fraction $\phi(z): H=\int_{0}^{\infty} \phi(z) d z$ and is directly related to the number of grains $N$ in the system (i.e., $N=6 H L W / \pi D^{3}$ ). The inclination angle is varied between $30^{\circ}$ and $50^{\circ}$ together with the restitution 


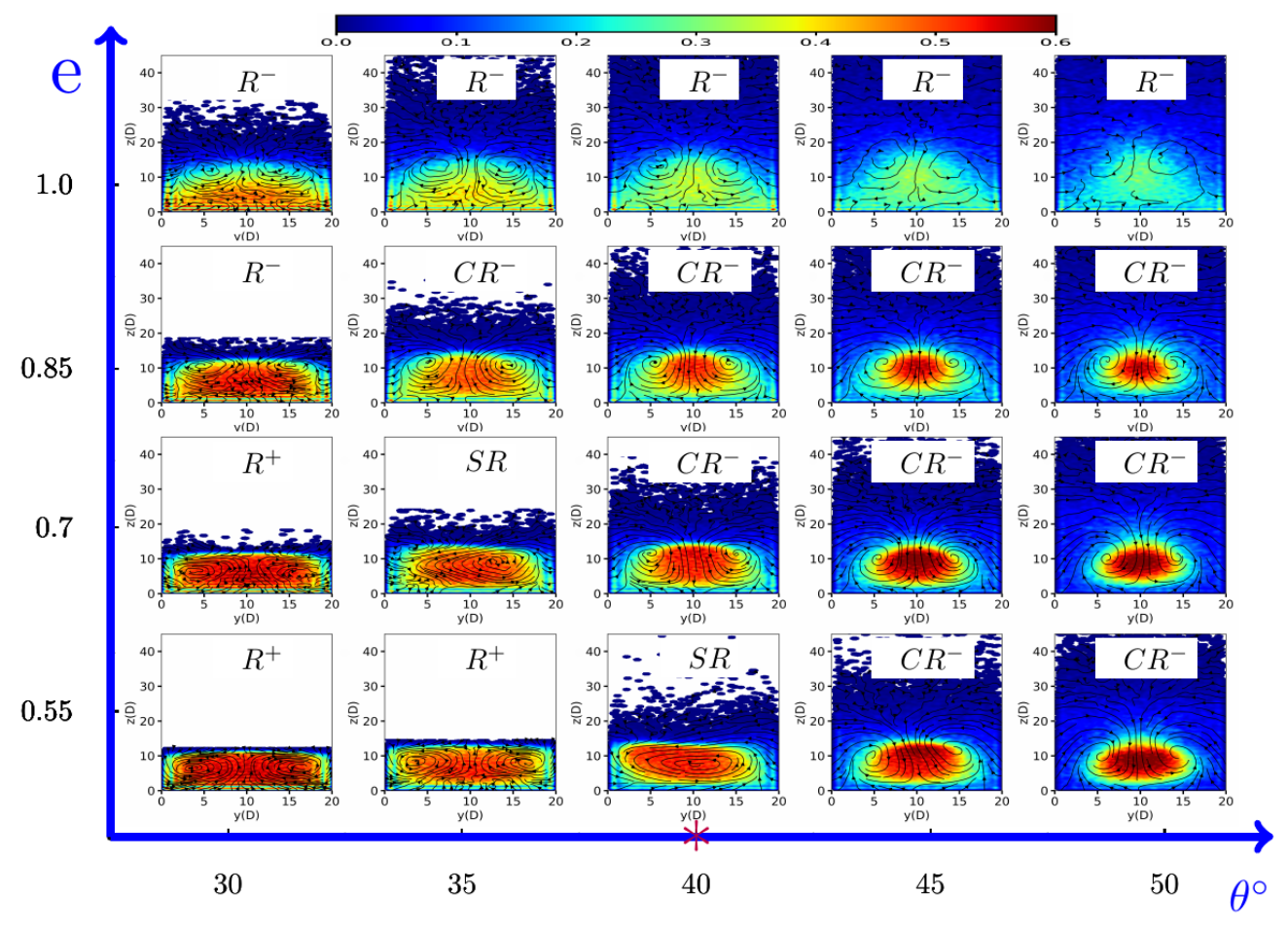

Figure 2: Cross-section of the flow showing the particle volume fraction together with the streamlines for various values of inclination angles and restitution coefficients $e$. A variety of different flow regimes are observed:(i) Dilute flow regime with a pair of longitudinal rolls $R^{-}$leading to a downward motion in the dense part of the flow (i.e., in the center of the cell); (ii) Dense flow regime with a pair of longitudinal vortices $R^{+}$leading to an upward motion in the denser part of the flow; (iii) Supported flow regime $C R^{-}$characterized by a dense core $C$ and a pair of longitudinal rolls $R^{-}$; (iv) flow regime $S R$ with a single longitudinal vortex.

coefficient $e$ from 1 down to 0.55. An illustration of the different flow regimes obtained when varying these parameters is given in Fig. 2.

Preliminary comments can be already made. Increasing the dissipation leads the flow to contract and thus to make it denser. This contraction triggers a transition in the flow regime: the dilute flow regime turns into a supported flow which is characterized by a dense core floating over a dilute and energetic gaseous phase. Additionally, the granular system exhibits secondary flows appearing as a pair of counter-rotative longitudinal vortices. The emergence of longitudinal vortices is a common feature of rapid flows $[1,6,10]$. In the dilute flow regime the pair of vortices (referred as $R^{-}$) induces a net downwards motion in the denser part of the flow (i.e., in center of the cell) and an upward motion in the more dilute region of the flow (i.e., at the side-walls). The transition from dilute to supported flow regime is accompanied with a reinforcement of the secondary flows. When the restitution coefficient is further decreased, we observe an other transition which modifies the rotation direction of the pair of longitudinal vortices: The downward motion is localized at the side-walls while the upward motion occurs at the center of the cell $\left(R^{+}\right.$flow regime in Fig. 2). This transition occurs via an intermediate state where one of the two vortices disappears. The remaining vortex extends over the whole width of the flow leading to a symmetry breaking ( $S R$ in Fig. 2).

\section{General features of the flow vs inelasticity}

We first investigate how the mean flow velocity is altered by the restitution coefficient. Unlike dense flows obtained at shallow angles, our rapid flows show a high sensitivity to the restitution coefficient as illustrated in Fig. 3. Surprisingly, the mean velocity increases with decreasing restitution coefficient. This velocity increase is more and more impressive as the angle of inclination increases. The more the system is dissipative, the faster it goes. We can note that for $\theta=30^{\circ}$ the increase of the flow velocity with increasing dissipation is much less important than for higher angles and saturates for values of $e$ smaller than 0.7. When $e$ is further decreased, the flow velocity even slightly decreases.

We can anticipate here that these outcomes result from the contraction of the flow, which leads to a decrease of the side-wall friction contribution to the over-whole resistive force. We plot in Fig. 4 the variation of the flowing height $h$, defined as the height below which one find $97 \%$ of the flowing material. The flow height is clearly decreasing with decreasing restitution coefficient. The in- 


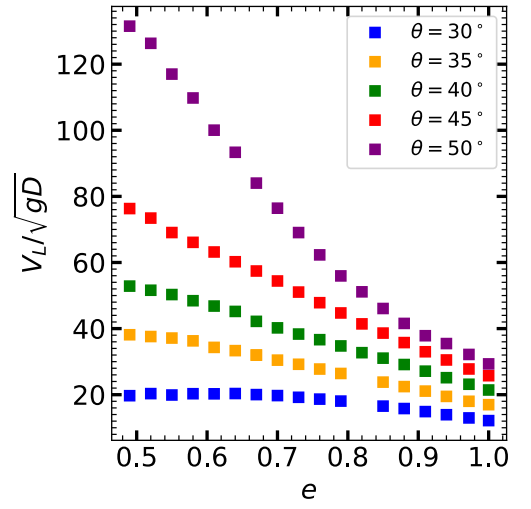

Figure 3: Mean flow velocity as a function of the restitution coefficient for various values of the inclination angle. The particle hold-up is $H=5 D$.

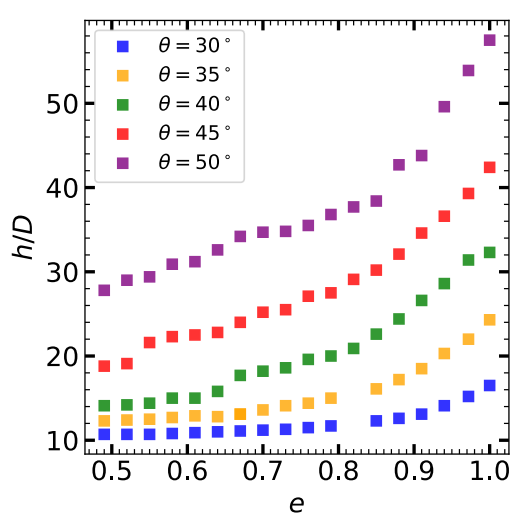

Figure 4: Flow height $h$ as a function of the restitution coefficient for various values of the inclination angle. The particle hold-up is $H=5 D$.

crease of the mean flow velocity seems to be directly correlated to the flow contraction. which can be interpreted as a consequence of the well-known clustering instability in granular gas $[11,12]$. The reverse trend observed for $\theta=30^{\circ}$ for high inelasticity results presumably from the fact the flow has reached a dense state where the contraction is no longer possible and the dissipation is dominated by particle-particle collisions rather than particle-wall collisions.

\section{Concentration, velocity and temperature profiles vs inelasticity}

It is instructive now to look more carefully about the flow structure and its change with decreasing restitution coefficient. We first present the vertical and transverse packing fraction profiles for a given angle $\theta=40^{\circ}$ and particle hold-up $H=5 D$ (see Fig. 5). For small inelasticity, the flow at $\theta=40^{\circ}$ is rather dilute and the packing fraction decreases monotonically as we move to the free surface. For higher inelasticity the flow undergoes a marked (a)

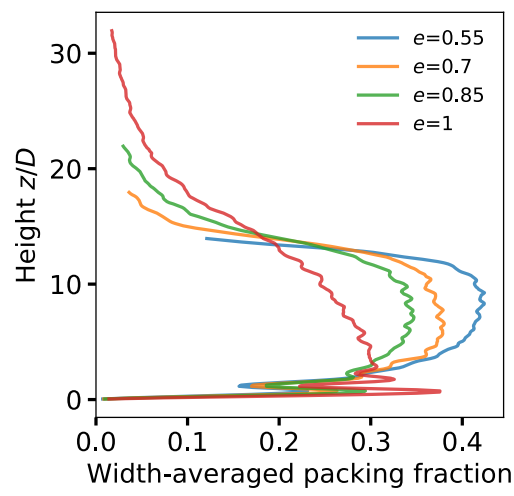

(b)

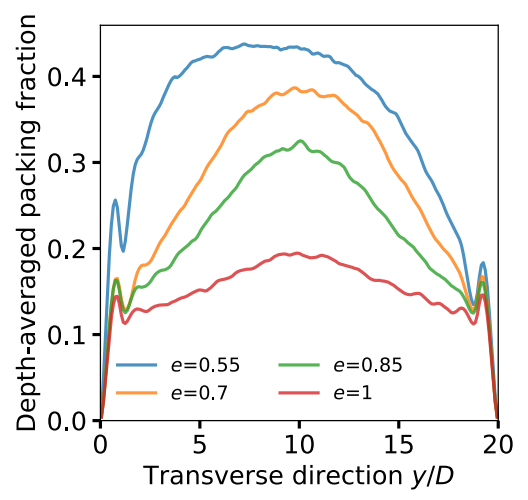

Figure 5: (a) Vertical packing fraction profiles for decreasing restitution coefficient. (b) Corresponding transverse packing fraction profiles. The inclination angle is $\theta=40^{\circ}$ and the particle hold-up is $H=5 D$.

transition where the volume fraction profile is inverted: it first increases and then decreases at larger height. A dense core emerges in the bulk flow with a high packing fraction which increases with decreasing restitution coefficient and is surrounded by a dilute atmosphere at the base and at the side-walls. This is the so-called "supported flow" which has been discovered by Brodu et al. in rapid flows [2]. Upon a further decrease of the restitution coefficient, we get another transition characterized by a change of the vortex pattern. The pair of vortices disappears and gives rise to a single longitudinal vortex which breaks the system symmetry, as can be seen in the transverse packing fraction profile (cf. Fig. 5b).

The influence of the inelasticity is also clearly seen on the vertical velocity profiles (see Fig. 6). For weak inelasticity (i.e., restitution coefficient close to 1), the velocity increases smoothly with increasing height and the slip velocity at the base is moderate. For increasing inelasticity, the slip velocity is increasing drastically. The shape of the velocity profile is also changed with a greater strain rate at the base and almost flat profile within the dense core. This is the classical feature of the supported regime with a dense core flowing as plug and moderately sheared in its interior. 


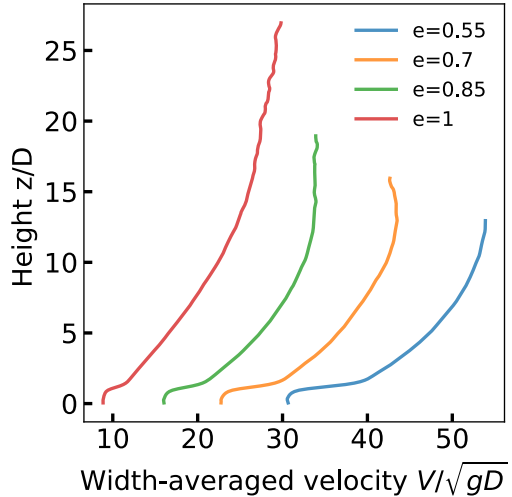

Figure 6: Vertical velocity profiles for decreasing restitution coefficient. The inclination angle is $\theta=40^{\circ}$ and the particle hold-up is $H=5 D$.

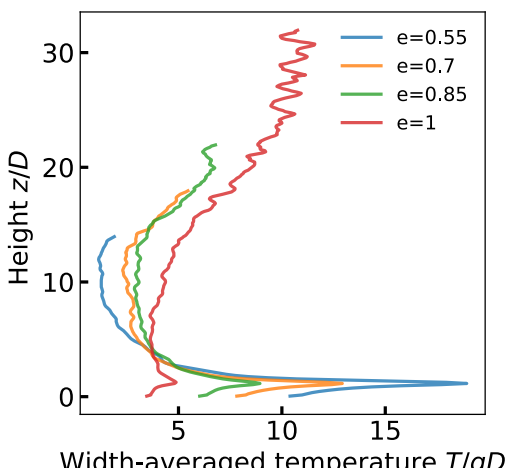

Figure 7: Vertical temperature profiles for decreasing restitution coefficient. The inclination angle is $\theta=40^{\circ}$ and the particle hold-up is $H=5 D$.

The temperature profiles are also very informative with regards to the role of inelasticity (see Fig. 7). As the inelasticity is increased, the temperature profile presents stronger temperature gradients. In particular, the base of the flow is very "hot" while the bulk flow is very "cold". This contrast of temperature increases with increasing inelasticity. The high temperature at the base is a consequence of both large slip velocity and rarefied atmosphere.

\section{Discussion and Conclusion}

Our simulation results have shown that the inelasticity plays an important role in the flow structure but also in the mean flow velocity in the context of rapid flows confined between frictional side walls. We found that inelastic flows runs faster than elastic ones. This counter-intuitive feature results in fact from the competition of two antagonist mechanisms. This first one already mentioned earlier is the effect of the flow contraction together with the creation of a dense core for increasing inelasticity. We believe that this is a direct consequence from the cluster instability in granular gas. The flow contraction reduces the area of friction with the side-wall and contribute to an increase of the mean flow velocity. The antagonist mechanism results from the increase of the effective side-wall friction coefficient when the flow velocity increases [4]. We showed in a recent paper that the effective wall friction is an increasing function of the Froude number. Thus for a given inclination angle and particle hold-up, the effective friction increases with increasing velocity. However, the increase of the effective friction coefficient is moderate in comparison with the contraction of the flow such that the latter prevails and is responsible for the mean velocity increase for increasing inelasticity.

The mean flow velocity increases with decreasing $e$ is one of the salient features concerning the role of the inelasticity in the context of rapid granular flows. We also mentioned the change of flow structure with increasing inelasticity, including the transition to the supported flow regime and the appearance of various patterns of longitudinal vortices. Further analysis are required to characterize and understand these flow transitions.

\section{Acknowledgements}

We acknowledge the support of the French Research National Agency through the project ANR-16-CE01-0005.

\section{References}

[1] N. Brodu, P. Richard, and R. Delannay, Phys. Rev. E 87(2), 022202 (2013)

[2] N. Brodu, R. Delannay, A. Valance, and P. Richard, J. Fluid Mech. 769, 218-228 (2015)

[3] S. Zhang, G. Yang, P. Lin, L. Chen and L. Yang, Eur. Phys. J. E 42, 40 (2019)

[4] Y. Zhu, R. Delannay, and A. Valance, Granul. Matter 22, 82 (2020)

[5] O. Hunger and N.R. Morgenstern, Géotechnique 34(3), 405-413 (1984)

[6] Y. Forterre and O. Pouliquen, J. Fluid Mech. 467, 361-387 (2002)

[7] A.J Holyoake and J.N. McElwaine, J. Fluid Mech. 710, 35-71 (2012)

[8] J. Heyman, P. Boltenhagen, R. Delannay, and A. Valance, EPJ Web Conf. 140, 03057 (2017)

[9] M.Y. Louge, and S.C. Keast, Phys. Fluids 13(5), 1213 (2001)

[10] T. Börzsönyi, R.E. Ecke and J.N. McElwaine, Phys. Rev. Lett. 103, 178302, (2009)

[11] I. Goldhirsch and G. Zanetti, Phys. Rev. Lett. 70, 1619 (1993)

[12] E. Opsomer, F. Ludewig, and N. Vandewalle, Eur. Phys. Lett. 99(4), 40001 (2012) 